\title{
Numerical Solution of Nonlinear Weakly Singular Fredholm-Volterra Integral Equations of the Second Kind by Using Sinc-Collocation Methods
}

\author{
Khosrow Maleknejad*, Azadeh Ostadi, Asyieh Ebrahimzadeh \\ School of Mathematics, Iran University of Science and Technology, Narmak, Tehran, 16846, Iran. \\ * Corresponding author. Tel.: +98 21732254 16; email: maleknejad@iust.ac.ir \\ Manuscript submitted February 13, 2016; accepted June 12, 2016. \\ doi: 10.17706/ijapm.2016.6.3.88-94
}

\begin{abstract}
In this paper, the efficient methods based on the Sinc approximation with the single exponential (SE) and double exponential (DE) transformations are presented to solve nonlinear Fredholm-Volterra integral equations with weakly singular kernel. Sinc approximation has considerable advantages. Some of them are the exponential convergence of an approximate solution and simply implementation, even in the presence of singularities. Properties of the SE-Sinc and DE-Sinc methods are utilized to reduce the problem to a set of algebraic equations. Finally, we give some numerical results that confirm efficiency and accuracy of the numerical schemes.
\end{abstract}

Key words: Nonlinear Fredholm-Volterra integral equation, Sinc approximation, weakly singular kernel.

\section{Introduction}

Many problems of engineering, contact problems in the theory of elasticity [1]-[3], mathematical physics and chemical reactions, such as heat conduction and crystal growth lead to singular integral equations. In this paper, we consider the weakly singular Fredholm-Volterra integral equations (F-VIEs) of the second kind of the form

$$
x(t)=g(t)+\int_{a}^{b} \frac{k_{1}(t, s, x(s))}{|t-s|^{1-\alpha}} d s+\int_{a}^{t} \frac{k_{2}(t, s, x(s))}{(t-s)^{1-\alpha}} d s, \quad a \leq t \leq b,
$$

where $0<\alpha<1 . k_{1}, k_{2}$ and $g$ are given functions, and $x$ is an unknown function to be determined. There is an increasing demand for studying singular integral equations and these problems of course cannot be solved explicitly. Therefore, it is important to find their approximate solutions by using some numerical methods. The product Nyström method has been devised to find numerical solution of singular F-VIEs in [4]. In [5], Fayazzadeh and Lotfi proposed collocation method to solve weakly singular F-VIEs.

A great deal of interest has been focused on applications of the Sinc methods. These methods have considerable advantages over classical methods that use polynomials as bases. For example, in the presence of singularities, these methods give an exponential convergence and accuracy than polynomial methods. Therefore, this paper describes procedures for solving nonlinear weakly singular F-VIE based on sinc approximation. The Sinc methods have been studied by many authors, e.g., Saadatmandi and Razzaghi [6], Okayama et al. [7], [8], Rashidinia [9], [10] and Maleknejad [10], [11]. 
The paper is organized as follows. In Section 2, we review some basic facts about the sinc approximation. Section 3 is devoted to solve (1) by using Sinc-collocation methods. As a result, a set of algebraic equations is achieved and the solution of the considered problem is introduced. In Section 4, we report our numerical findings and demonstrate validity of the proposed schemes by considering numerical examples.

\section{Sinc Function and Basic Definition}

In this section, we will review Sinc function properties. These are discussed thoroughly in [12], [13]. Originally, Sinc approximation for a function $f$ is expressed as

$$
f(x) \approx \sum_{j=-N}^{N} f(j h) S(j, h)(x), \quad x \in \mathbb{R}
$$

To construct approximation on the interval $L=(a, b)$, which are used in (1), we consider the tanh transformation (and its inverse)

$$
\begin{gathered}
\emptyset^{S E}(x)=\frac{b-a}{2} \tanh \left(\frac{x}{2}\right)+\frac{b+a}{2}, \\
\left\{\varnothing^{S E}\right\}^{-1}(t)=\log \left(\frac{t-a}{b-t}\right) .
\end{gathered}
$$

Interpolation formula for $f(t)$ over $(a, b)$ is

$$
f(t) \approx \sum_{j=-N}^{N} f\left(\phi^{S E}(j h)\right) S(j, h)\left(\left\{\phi^{S E}\right\}^{-1}(t)\right)
$$

Sinc approximation can be applied to definite integration based on the function approximation described above; it is called the Sinc quadrature. Where

$$
\int_{a}^{b} f(s) d s \approx h \sum_{j=-m}^{n} f\left(\phi^{S E}(j h)\right)\left\{\phi^{S E}\right\}^{\prime}(j h) .
$$

The following theorems give us an error bound for the SE-Sinc approximation and quadrature.

Theorem 1 ([12]): Let $f \in L_{\alpha}\left(\phi^{S E}\left(D_{d}\right)\right)$ for $d$ with $0<d<\pi$. Let also $N$ be a positive integer, and $h$ be given by the formula

$$
h=\sqrt{\frac{\pi d}{\alpha N}}
$$

Then there exists a constant $C$ independent of $N$, such that

$$
\left|f(t)-\sum_{j=-N}^{N} f\left(\varnothing^{S E}(j h)\right) S(j, h)\left(\left\{\emptyset^{S E}\right\}^{-1}(t)\right)\right| \leq C \sqrt{N} \exp (-\sqrt{\pi d \alpha N})
$$

Theorem 2 ([12]): Let $(f Q) \in L_{\alpha}\left(\phi^{S E}\left(D_{d}\right)\right)$ for $d$ with $0<d<\pi$. Suppose that $N$ is a positive integer and $h$ is selected by (3). Then there exists a constant $C$ independent of $N$, such that 


$$
\left|\int_{a}^{b} f(s) d s-h \sum_{j=-m}^{n} f\left(\varnothing^{S E}(j h)\right)\left\{\varnothing^{S E}\right\}^{\prime}(j h)\right| \leq \mathrm{C} \exp (-\sqrt{\pi d \alpha N})
$$

Also, double exponential transformation can be used

$$
\begin{gathered}
\emptyset^{D E}(x)=\frac{b-a}{2} \tanh \left(\frac{\pi}{2} \sinh (x)\right)+\frac{b+a}{2} \\
\left\{\varnothing^{D E}\right\}^{-1}(t)=\log \left(\frac{1}{\pi} \log \left(\frac{t-a}{b-t}\right)+\sqrt{1+\left(\frac{1}{\pi} \log \left(\frac{t-a}{b-t}\right)\right)^{2}}\right) .
\end{gathered}
$$

The following theorems describe the accuracy of DE-Sinc method.

Theorem 3 ([14]): Let $f \in L_{\alpha}\left(\phi^{D E}\left(D_{d}\right)\right)$ for $d$ with $0<d<\frac{\pi}{2}$. Let also $N$ be a positive integer, and $h$ be given by the formula

$$
h=\frac{\log (2 d N / \alpha)}{N}
$$

Then there exists a constant $C$ which is independent of $N$, such that

$$
\left|f(t)-\sum_{j=-N}^{N} f\left(\emptyset^{D E}(j h)\right) S(j, h)\left(\left\{\varnothing^{D E}\right\}^{-1}(t)\right)\right| \leq C \exp \left(\frac{-\pi d N}{\log \left(\frac{2 d N}{\alpha}\right)}\right)
$$

Such a function is required to be zero at the endpoints, $t=a$ and $t=b$, which seems to be an impractical assumption. In order to handle more general cases, we introduce the translated function

$$
\Gamma[f](t)=f(t)-\left[\left(\frac{b-t}{b-a}\right) f(a)+\left(\frac{t-a}{b-a}\right) f(b)\right] .
$$

Theorem 4 ([8]): Assume that $(f Q) \in L_{\alpha}\left(\emptyset^{D E}\left(D_{d}\right)\right)$ for $d$ with $0<d<\frac{\pi}{2}$. Suppose that $N$ is a positive integer and $h$ is selected by (6). Then there exists a constant $C$ independent of $N$, such that

$$
\left|\int_{a}^{b} f(s) d s-h \sum_{j=-m}^{n} f\left(\varnothing^{D E}(j h)\right)\left\{\emptyset^{D E}\right\}^{\prime}(j h)\right| \leq \mathrm{C} \exp \left(\frac{-2 \pi d N}{\left.\log \frac{2 d N}{\alpha}\right)}\right)
$$

\section{Sinc-Collocation Methods}

In this Section, illustrate how the Sinc methods may be used to replace (1) by a system of nonlinear algebraic equations. Equation (1) can be written in the operator form $x=\kappa[x]+\boldsymbol{g}$ where

$$
\kappa[x](t)=\int_{a}^{b} \frac{k_{1}(t, s, x(s))}{|t-s|^{1-\alpha}} d s+\int_{a}^{t} \frac{k_{2}(t, s, x(s))}{(t-s)^{1-\alpha}} d s
$$

\subsection{SE-Sinc Scheme}

A Sinc approximation $x_{N}^{S E}$ to the solution $x \in M_{\alpha}\left(\phi^{S E}\left(D_{d}\right)\right)$ of above equation is described in this part. 
The function can be accurately approximated as

$$
\Gamma[x](t) \approx \sum_{j=-N}^{N} \Gamma[x]\left(\phi^{S E}(j h)\right) S(j, h)\left(\left\{\phi^{S E}\right\}^{-1}(t)\right) .
$$

So, the approximate solution $x$ is considered that has the form

$$
P_{N}^{S E}[x](t)=x(a) \frac{b-t}{b-a}+\sum_{j=-N}^{N} \Gamma[x]\left(\phi^{S E}(j h)\right) S(j, h)\left(\left\{\phi^{S E}\right\}^{-1}(t)\right)+x(b) \frac{t-a}{b-a},
$$

where $h$ is given by (3). Now, we can obtain the convergence theorem corresponding to Theorem 1 .

Theorem 5: Let $f \in M_{\alpha}\left(\phi^{S E}\left(D_{d}\right)\right)$ for d with $0<d<\pi$. Let also $N$ be a positive integer, and $h$ be given by (3). Then there exists a constant $C^{S E}$ independent of $N$, such that

$$
\max _{a \leq t \leq b}\left|f(t)-P_{N}^{S E}[f]\right| \leq C^{S E} \sqrt{N} \exp (-\sqrt{\pi d \alpha N}) .
$$

There are $(2 N+3)$ unknown coefficients on the right-hand side of $(10)$ that should be determined. So, the approximate solution $x_{N}^{S E}$ has a form like

$$
x_{N}^{S E}=c_{-N-1} \frac{b-t}{b-a}+\sum_{j=-N}^{N} c_{N} S(j, h)\left(\left\{\phi^{S E}\right\}^{-1}(t)\right)+c_{N+1} \frac{t-a}{b-a},
$$

In order to determine unknowns, we apply the collocation method. Summarily, by setting $t=t_{j}^{S E}$ where

$$
t_{j}^{S E}=\left\{\begin{array}{cr}
a & j=-N-1, \\
\phi^{S E}(j h) & j=-N, \ldots, N, \\
b & j=N+1,
\end{array}\right.
$$

in (12) and then by substituting $x_{N}^{S E}$ into (1) and applying the collocation method to it, we obtain the following system of nonlinear equations with $(2 N+3)$ unknowns $c_{j}, j=-N-1, \ldots, N+1$ :

$$
\begin{gathered}
x_{N}^{S E}\left(t_{i}^{S E}\right)-\int_{a}^{b} k_{1}\left(t_{i}^{S E}, s, x_{N}^{S E}(s)\right)\left|t_{i}^{S E}-s\right|^{\alpha-1} d s-\int_{a}^{t} k_{2}\left(t_{i}^{S E}, s, x_{N}^{S E}(s)\right)\left(t_{i}^{S E}-s\right)^{\alpha-1} d s= \\
g\left(t_{i}^{S E}\right) .
\end{gathered}
$$

Since the SE-Sinc quadrature does not allow any singularity in the target interval, we split the first integral into two parts at $s=t_{j}^{S E}$. In order to approximate (13) we employ (5) and then

$$
g\left(t_{i}^{S E}\right)=x_{N}^{S E}\left(t_{i}^{S E}\right)-\kappa_{N}^{S E}\left[x_{N}^{S E}\right]\left(t_{i}^{S E}\right),
$$

where $\kappa_{N}^{S E}$ is defined by

$$
\begin{aligned}
\kappa_{N}^{S E}[x](t)=h \sum_{j=-M}^{N}\left(t-\phi_{a, t}^{S E}(j h)\right)^{\alpha-1} k_{1}\left(t, \phi_{a, t}^{S E}(j h), x\left(\phi_{a, t}^{S E}(j h)\right)\right)\left\{\phi_{a, t}^{S E}\right\}^{\prime}(j h) \\
+h \sum_{j=-N}^{M}\left(\phi_{t, b}^{S E}(j h)-t\right)^{\alpha-1} k_{1}\left(t, \phi_{t, b}^{S E}(j h), x\left(\phi_{t, b}^{S E}(j h)\right)\right)\left\{\phi_{t, b}^{S E}\right\}^{\prime}(j h) \\
+h \sum_{j=-M}^{N}\left(t-\phi_{a, t}^{S E}(j h)\right)^{\alpha-1} k_{2}\left(t, \phi_{a, t}^{S E}(j h), x\left(\phi_{a, t}^{S E}(j h)\right)\right)\left\{\phi_{a, t}^{S E}\right\}^{\prime}(j h)
\end{aligned}
$$


It should be pointed out that $M$ is set by $M=[\alpha N]$. The above nonlinear system consists of $2 N+3$ equations with $2 N+3$ unknown $\left\{c_{j}\right\}_{-N-1}^{N+1}$. This system can be rewritten as $\mathcal{F}^{S E}\left(x_{N}^{S E}\right)=0$, where $\mathcal{F}^{S E}: \mathbb{R}^{2 N+3} \rightarrow \mathbb{R}^{2 N+3}$ with $\mathcal{F}^{S E}\left(x_{N}^{S E}\right)=x_{N}^{S E}-\kappa_{N}^{S E}\left[x_{N}^{S E}\right]-g$. By solving this nonlinear system by Newton's method, we can obtain the approximate solution $x_{N}^{S E}$.

\subsection{DE-Sinc Scheme}

In this case, we assume that the solution of (1) belongs to $M_{\alpha}\left(\phi^{D E}\left(D_{d}\right)\right)$. Similar to the SE-Sinc method, by Theorem 3, the following theorem can be inferred.

Theorem 6: Let $f \in M_{\alpha}\left(\phi^{D E}\left(D_{d}\right)\right)$ for $d$ with $0<d<\frac{\pi}{2}$. Let also $N$ be a positive integer, and $h$ be given by (6). Then there exists a constant $C^{D E}$ which is independent of $N$, such that

$$
\max _{a \leq t \leq b}\left|f(t)-P_{N}^{D E}[f]\right| \leq C^{D E} \exp \left(\frac{-\pi d N}{\log \left(\frac{2 d N}{\alpha}\right)}\right)
$$

To apply the collocation method, set $t=t_{j}^{D E}, j=-N-1, \ldots, N+1$ are Sinc grid points

$$
t_{j}^{D E}=\left\{\begin{array}{cr}
a & j=-N-1, \\
\phi^{D E}(j h) & j=-N, \ldots, N \\
b & j=N+1
\end{array}\right.
$$

It should be noted that in DE case $M$ is set by $M=N+\left[\frac{\log (\alpha)}{h}\right]$.

\section{Numerical Examples}

In order to illustrate the performance of the Sinc methods in solving weakly singular F-VIE and justify the accuracy and efficiency of the presented methods, we consider the following examples.

Example 1: Consider the following weakly singular F-VIE

$$
x(t)=\sqrt{t}-\frac{\pi t^{3 / 2}}{2 \sqrt{t}}-\frac{2}{3}\left(\sqrt{1-t}+2 t \sqrt{1-t}+2 t^{\frac{3}{2}}\right)+\int_{0}^{1} \frac{(s(s))^{2}}{|t-s|^{\frac{1}{2}}} d s+\int_{0}^{t} \frac{x(s)}{(t-s)^{1 / 2}} d s, \quad t \in[0,1] .
$$

where the exact solution is $x(t)=\sqrt{t}$. Fig. 1 show maximum absolute errors corresponding to $\mathrm{SE}$ and DE-Sins methods. It shows that DE-Sinc method is more accurate than SE-Sinc method.

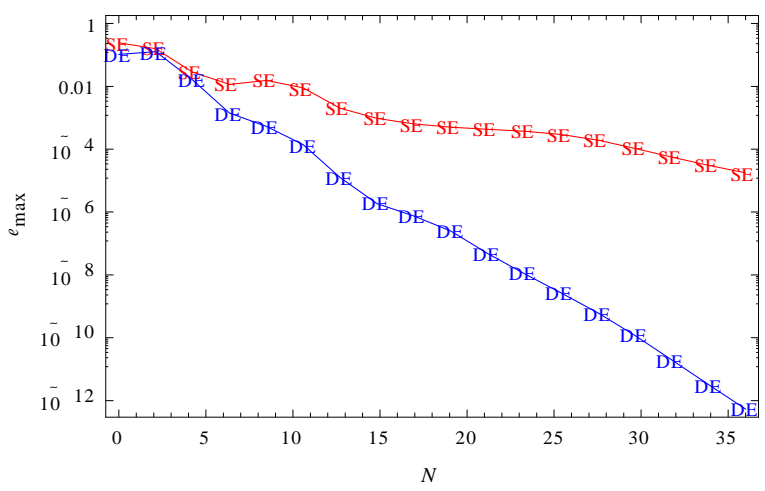

Fig. 1. The SE and DE-Sinc results for example 1.

Example 2: Consider the problem 


$$
x(t)=g(t)+\int_{0}^{1} \frac{(s(s))^{2}}{|t-s|^{\frac{1}{4}}} d s+\int_{0}^{t} \frac{\sin (x(s))}{(t-s)^{1 / 4}} d s, \quad t \in[0,1] .
$$

where $g(t)$ is obtained so that $x(t)=\sqrt{t}$ is the solution. We list the absolute errors for several selected values of $N$ for SE and DE-Sinc methods in Tables 1 and 2. Tables show that the convergence rate of the DE-Sinc method is much faster than the SE-Sinc scheme. Moreover, Fig. 2 shows maximum absolute errors for each method. This figure shows that DE-Sinc method is more accurate than SE-Sinc method.

Table 1. Absolute Errors of the SE-Sinc Method for Example 2

\begin{tabular}{lllll}
\hline \hline $\mathrm{t}$ & $\mathrm{N}=5$ & $\mathrm{~N}=15$ & $\mathrm{~N}=25$ & $\mathrm{~N}=35$ \\
\hline 0.1 & $1.66 \mathrm{E}-3$ & $4.86 \mathrm{E}-6$ & $4.73 \mathrm{E}-7$ & $1.91 \mathrm{E}-8$ \\
0.3 & $6.94 \mathrm{E}-4$ & $1.07 \mathrm{E}-5$ & $9.22 \mathrm{E}-7$ & $6.81 \mathrm{E}-8$ \\
0.5 & $5.16 \mathrm{E}-4$ & $1.07 \mathrm{E}-5$ & $6.08 \mathrm{E}-7$ & $5.79 \mathrm{E}-8$ \\
0.7 & $2.49 \mathrm{E}-3$ & $1.91 \mathrm{E}-5$ & $4.74 \mathrm{E}-7$ & $3.25 \mathrm{E}-8$ \\
0.9 & $7.15 \mathrm{E}-4$ & $1.70 \mathrm{E}-5$ & $7.19 \mathrm{E}-7$ & $3.77 \mathrm{E}-8$ \\
\hline \hline
\end{tabular}

Table 2. Absolute Errors of the DE-Sinc Method for Example 2

\begin{tabular}{lllll}
\hline \hline $\mathrm{t}$ & $\mathrm{N}=5$ & $\mathrm{~N}=15$ & $\mathrm{~N}=25$ & $\mathrm{~N}=35$ \\
\hline 0.1 & $5.81 \mathrm{E}-3$ & $2.69 \mathrm{E}-7$ & $7.20 \mathrm{E}-11$ & $2.79 \mathrm{E}-14$ \\
0.3 & $7.83 \mathrm{E}-3$ & $4.38 \mathrm{E}-9$ & $8.10 \mathrm{E}-11$ & $1.09 \mathrm{E}-14$ \\
0.5 & $3.58 \mathrm{E}-3$ & $4.72 \mathrm{E}-8$ & $8.22 \mathrm{E}-12$ & $2.78 \mathrm{E}-15$ \\
0.7 & $1.40 \mathrm{E}-2$ & $7.24 \mathrm{E}-8$ & $7.33 \mathrm{E}-11$ & $1.41 \mathrm{E}-14$ \\
0.9 & $1.15 \mathrm{E}-3$ & $2.18 \mathrm{E}-7$ & $6.55 \mathrm{E}-11$ & $2.61 \mathrm{E}-14$ \\
\hline \hline
\end{tabular}

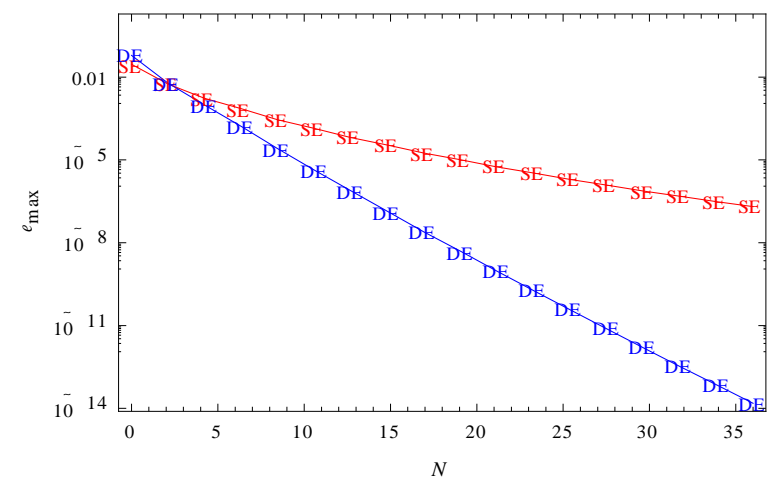

Fig. 2. The SE and DE-Sinc results for example 2.

\section{References}

[1] Lifanov, I. K., Poltavskii, L. N., \& Vainikko, G. M. (2004). Hypersingular Integral Equations and Their Applications. CRC Press

[2] Aleksandrov, V. M., \& Kovalenko, E. V. (1983). On a method of obtain spectral relationships for integral operators of mixed problem of mechanics of continuous media. Applied Mathematics and Mechanics, $4 b(b), 825-832$.

[3] Semetanian, B. J. (1991). On an integral equation for axially symmetric problem in the case of an elastic body containing an inclusion. Journal of Applied Mathematics and Mechanics, 55(3), 371-375.

[4] El-Kalla, I. L., \& Bugami, A. M. (2011). Fredholm-Volterra integral equation with a generalized singular kernel and its numerical solutions. International Journal of Research and Reviews in Applied Sciences, 6(3), 341-352.

[5] Fayazzadeh, S., \& Lotfi, M. (2011). Collocation method for Fredholm-Volterra integral equations with weakly kernels. International Journal of Mathematical Modelling \& Computations, 1, 59-68. 
[6] Saadatmandi, A., \& Razzaghi, M. (2007). The numerical solution of third-order boundary value problems using Sinc collocation method. Communications in Numerical Methods in Engineering, 23, 681-689.

[7] Okayama, T., Matsuo, T., \& Sugihara, M. (2010). Sinc-collocation methods for weakly singular Fredholm integral equations of the second kind. Journal of Computational and Applied Mathematics, 234, 1211-1227.

[8] Okayama, T., Matsuo, T., \& Sugihara, M. (2009)., Error estimates with explicit constants for Sinc approximation, Sinc quadrature and Sinc indefinite integration(Mathematical Engineering Technical Reports 2009-01). The University of Tokyo.

[9] Rashidinia, J., \& Zarebnia, M. (2007). New approach for numerical solution of Hammerstein integral equations. Applied Mathematics and Computation, 185, 147-154.

[10] Rashidinia, J., Maleknejad, K., \& Taheri, N. (2013). Sinc-Galerkin method for numerical solution of the Bratu's problems. Numerical Algorithms, 62, 1-11.

[11] Maleknejad, K., Mollapourasl, R., \& Ostadi, A. (2015). Convergence analysis of Sinc-collocation methods for nonlinear Fredholm integral equations with a weakly singular kernel. Journal of Computational and Applied Mathematics, 278, 1-11.

[12] Stenger, F. (1993). Numerical Methods Based on Sinc and Analytic Functions. New York: Springer-Verlag.

[13] Lund, J., \& Bowers, K. (1992). Sinc methods for quadrature and differential equations. Philadelphia. PA.: SIAM.

[14] Sugihara, M., \& Matsua, T. (2004). Recent developments of the Sinc numerical methods. Journal of Computational and Applied Mathematics, 164-165, 673-689.

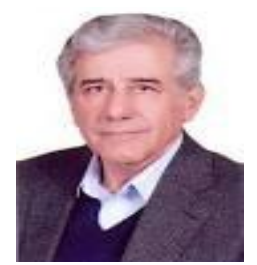

Khosrow Maleknejad obtained his MSc degree in applied mathematics from Tehran University, Iran, in 1972 and received his PhD degree in applied mathematics in numerical analysis area from the University of Wales, Aberystwyth, UK in 1980. In September 1972, he joined the School of Mathematics at Iran University of Science and Technology (IUST), Narmak, Tehran, Iran where he was previously lecturer, assistant professor and associate professor at IUST. He has been a professor since 2002 at IUST. He was a visiting professor at the University of California at Los Angeles in 1990. His research interests include numerical analysis in solving ill-posed problems and solving Fredholm and Volterra integral equations. He has authored as a Editor-in-Chief of the International Journal of Mathematical Sciences, which publishers by Springer. He is a member of the AMS.

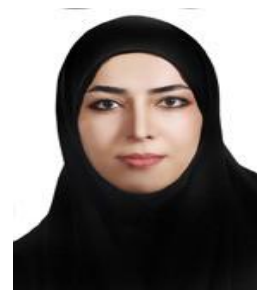

Azadeh Ostadi is a Ph.D student in the Department of Applied Mathematics at Iran University of Science and Technology, Tehran, Iran. She obtained M.Sc in mathematics in 2011 at Shahid Rajaee Teacher Training University, Tehran, Iran. She also obtained Bs.C in mathematics at Damghan University, Semnan, Iran. Her scientific interests include numerical analysis in solving integral equations with weakly singular kernel and Sinc approximation.

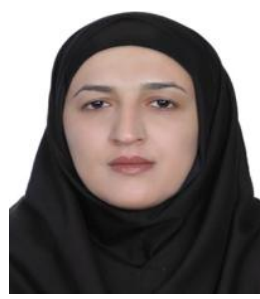

Asyieh Ebrahimzadeh is a Ph.D student in the Department of Applied Mathematics at Iran University of Science and Technology, Tehran, Iran. She obtained M.Sc in mathematics in 2009 at Khaje Nasir Toosi University of Technology, Tehran, Iran. She also obtained Bs.C in mathematics at ValiAsr University of Rafsanjan, Kerman, Iran. Her scientific interests include: optimal control, wavelets, integral equations and orthogonal functions. 